\title{
OVERVIEW OF CHINESE AND AMERICAN MARINE AIRBORNE LIDAR
}

\author{
Yizhi Tan ${ }^{1,2}$, Guoqing Zhou ${ }^{1}$,Xiang Zhou ${ }^{1,2,3,{ }^{*}}$, Jiandong Wei ${ }^{1,2}$, Jinlong Chen ${ }^{1,2}$, Haocheng Hu ${ }^{1,2}$ \\ ${ }^{1}$ Guangxi Key Laboratory of Spatial Information and Geometrics, Guilin University of Technology, No. 12 Jian'gan Road, Guilin, \\ Guangxi 541004, China - gzhou@glut.edu.cn \\ ${ }^{2}$ College of Mechanical and Control Engineering, Guilin University of Technology, No. 12 Jian'gan Road, Guilin, Guangxi 541004, \\ China - (1020180612, zqx0711)@glut.edu.cn \\ ${ }^{3}$ School of Microelectronics, Tianjin University, No. 92 Weijin Road, Tianjin 300072, China
}

KEY WORDS: Marine Airborne Lidar, Seawater Sounding, Lidar Development, Technology Improvement, Hardware parameters, Technology gap

\begin{abstract}
:
As one of the most popular surveillance technologies in the 21 st century, Lidar is applied in commercial, military and civilian applications. paper mainly introduces the development process of Chinese and American marine lidar detection technology, compares the hardware technical parameters of marine airborne laser lidar between China and the United States, and summarizes China's current airborne lidar detection technology and developed countries. Find the gap between developed countries. It introduces the most important application of this technology, and its contribution to the development of science and technology. Finally, summarize the various problems encountered in the development of the technology, analyse the reasons, and look forward to the future development trend.
\end{abstract}

*Corresponding author: Xiang Zhou; Email: zqx0711@glut.edu.cn 


\section{INTRODUCTION}

China is located in the eastern part of the Asian continent and faces the Pacific Ocean. Faced with such a vast ocean and abundant marine resources, it plays an important role to explore near-shore water depth. Although the detection range of airborne marine lidar is not as long as sonar technology, its search efficiency and detection point density are much higher than sonar, and in seawater hydrographic survey, underwater submarine detection, mine detection, fish detection, and ocean Many areas (Chen et al.,1998).Airborne lidar is an active measurement technique that can quickly and directly capture the water depths of shallow seas, such as shallow seas, island reefs, reefs and ships that cannot be reached smoothly. It is considered to be a potential new ground observation in the field of marine surveying and mapping technology. Airborne laser ocean sounding technology is a system that integrates optics, mechanics and electricity. The lidar bathymetry system is divided into two kinds: single-band airborne laser bathymetry and dual-band airborne laser bathymetry. The single-band blue-green laser with $532 \mathrm{~nm}$ wavelength is used as the laser emission source, and the double-band blue-green laser with $1064 \mathrm{~nm}$ near infrared and $532 \mathrm{~nm}$ is selected as the laser emission source. Dual-band airborne laser bathymetry system emits two kinds of lasers at the same time to the sea surface (Zhang et al.,2015). Infrared light penetrates poorly and will be reflected back when it reaches the water surface. Blue-green laser can penetrate the sea surface to shallow water and deep water, and will be reflected back when it reaches a certain depth.

Our research team won a key project of innovation-driven development in Guangxi in 2018. The purpose of this project is to develop an airborne laser high precision three-dimensional seabed measurement instrument (LiDAR). The principle and structure of the instrument are shown in Fig. 1. My task is mainly to study the blue and green light detection circuit. In this paper, I summarize the development process of underwater airborne lidar in China and the United States, so that you can have a clearer understanding of lidar bathymetry technology.

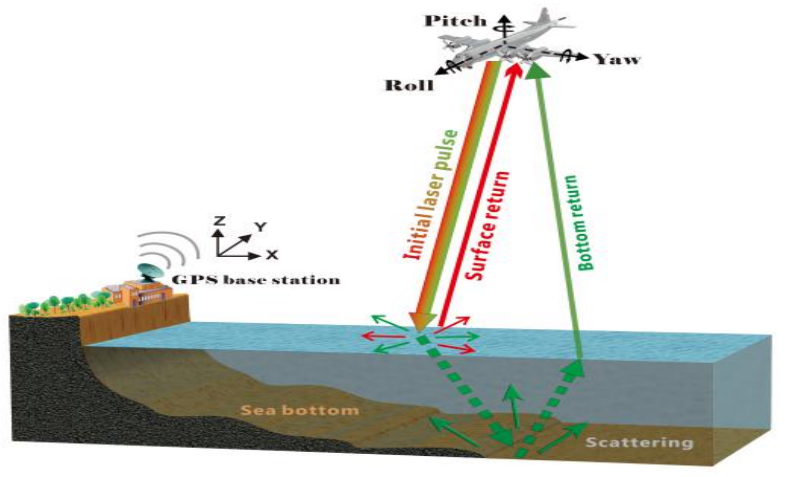

Fig 1. Dual LiDAR working diagram

\section{DEVELOPMENT OF MARINE AIRBORNE LIDAR}

In the 1960s, the world's first laser lidar came out (Penny et al.,1989). Airborne lidar technology, it has been recognized by many scientists. Until the 1920s, the research and application of airborne lidar have made considerable progress. The traditional aerial photogrammetry operation is still the main force in the field of remote sensing, the airborne lidar detection technology is likely to be in the future. Become one of the mainstreams. In the 1990s, the airborne lidar system entered the commercial stage and was widely used in coastal erosion monitoring, surveying and mapping. With the rapid development of high-precision GPS positioning technology and inertial navigation technology, the three-dimensional position of direct and fast acquisition of large-scale ground targets from the air has a corresponding technical basis, and the laser scanning system has gradually replaced the airborne section measurement system. The airborne laser scanning system was developed by the American laboratory in 1992 and the results were published later (Chang et al.,2002). The International Society for Photogrammetry and Remote Sensing presented a manufacturer to drill an airborne laser scanning system at an international conference in Vienna. Later, more manufacturers and more service providers entered the field of airborne laser scanning systems.

The United States have carried out the ocean Lidar exploration. China's Institute of Remote Sensing Applications of the Chinese Academy of Sciences developed the Chinese airborne laser three-dimensional imager with the support of the National 863 Program in the late 1990s, and successfully applied the application(Li et al.,2000).After 2010, Beijing Siwei Vision Company launched a commercialized airborne laser scanning system and vehicle laser scanning system to realize the localization of laser scanning equipment. At present, with the expansion of the demand for high-precision topographic surveying and mapping in the traditional industry, the application of domestic laser lidar technology is entering a new era of vigorous development. Taking Huazhong University of Science and Technology as an example, they successfully developed a CAYT sounding system and conducted a sounding test in the South China Sea in 1996, with a depth of 80 to $90 \mathrm{~m}$ (Chang et al.,2002). The Shanghai Institute of Optics and Fine Mechanics and the China Ocean University have also developed a variety of airborne marine laser lidar. Which measures sea depth and temperature.

The paper mainly describes the development of underwater lidar between the United States and China:

\subsection{American Marine Airborne Lidar}

The United States was the first country to study marine lidar technology. It established the world's first sounding system as early as 1968(Zhou et al.,2018). Based on theoretical knowledge and experimental platform, a water depth was developed in 1971. The detection laser lidar, the ALB system uses a green laser $532 \mathrm{~nm}$ that can penetrate a certain depth of water (Steinvall et al.,1998). The depth of sounding is about 10 meters. In the late 1970 s, a new detection system with high-speed data recording capability was developed. The laser used was a helium-neon laser with a depth of about 10 meters. However, these systems were developed only for laboratory use. In 1988, the US "Roberts" frigate was hit by a mine in the Arabian Gulf and suffered heavy losses. Since then, Kaman Aerospace has developed a mine detection laser lidar called "Magic Light". As shown in the following figure2. 


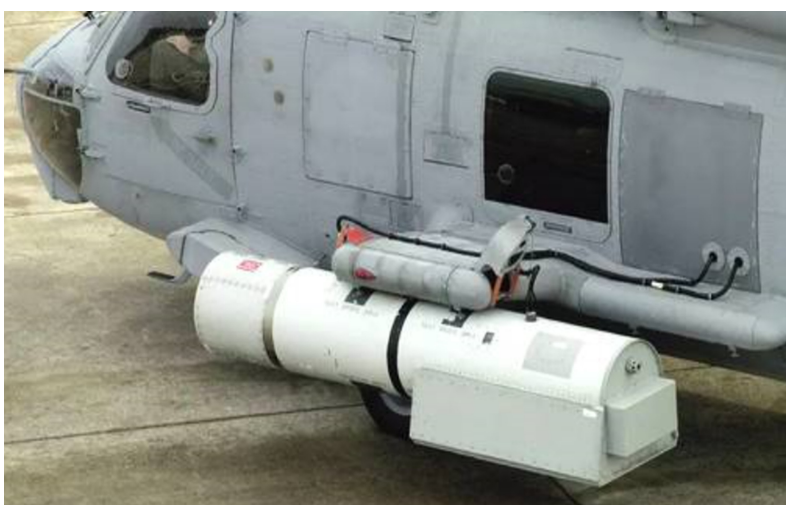

Figure 2. Magic light Airborne Laser Mine Detection System Installed on MCH-101 Sea Devil Helicopter (courtesy: https://jmqmil.sina.cn/ifeng/doc-ihhacrce9339710.d.html)

The lidar is equipped with a blue-green laser, it can quickly detect underwater targets and automatically implement target classification and location. During the 1991 Gulf War, Magic Lantern Lidar was deployed to the Gulf and successfully found mines and mine anchors (Lee et al.,2010). At present, "Magic Lamp" lidar has been equipped with helicopters of naval aviation forces. The airborne mine detection system developed by Northrop Company in the United States has automatic, real-time detection function and three-dimensional positioning ability. It has high positioning resolution and it lasts all day.it still can work at a height of $120-460 \mathrm{~m}$ above the sea surface, the nominal working height is $460 \mathrm{~m}$, but the resolution and signal-to-noise ratio are relatively high at low altitude, and the field of view is limited. The depth of detection was initially set at 12 to $61 \mathrm{~m}$ in shallow water (Von et al.,1995). The airborne mine detection system was developed around 2005 and was finally installed on the H-60 helicopter. The ATD-111 lidar is another mine search laser lidar of the US Navy. Developed by Sanders, the lidar is a pod-borne system that can be mounted on the SH-60 Sea hawk helicopter (Chen et al.,1998). Mechanical synchronous scanning SM2000 underwater laser imaging system produced by the US Westinghouse for the US Navy. The imaging distance is longer than that of a normal underwater camera, and the effective field of view can reach $70^{\circ}$. It can be distinguished at a distance of $30 \mathrm{~m}$. The effective field of view of the system is approximately five times that of the gating technology, and the imaging quality is better than the distance gating. After the 1970s, Linda Mullen and Alan Laws, engineers of the US Naval Air Warfare Aviation Division, developed and elaborated a new method to improve underwater imaging performance in a drowning environment (Wang et al.,2006). The underwater optical method encodes the lidar signal to improve image quality, thereby enhancing the fleet's ability to move.it can work 24 hours, adopt oval scanning mode, the front part of the scanning shows the colour coding depth of the scanning point, and the rear part turns the real colour into flashing white according to the correlation of scanning points, showing suspicious underwater targets.

\subsection{Chinese Marine Airborne Lidar}

China has started research on airborne laser sounding and underwater target detection since the late 1980s. In 2003, Huazhong University of Science and Technology developed China's first airborne lidar underwater detection test system, it using Nd:YAG, dual-wavelength laser, repetition frequency of $100 \mathrm{~Hz}$, and scanning, high-speed data storage and Recording function, in the sea trial in 1996, detected a submarine echo signal with a water depth of $80-90 \mathrm{~m}$ (Zhao et al.,2010).In September 2003, the State Council approved the launching of the "China's Offshore Marine Comprehensive Survey and Assessment" special project, mainly to find out the background of China's island coastal zone (Ren et al.,2005).This special experiment obtained massive survey data, formed China's first set of island coastal zone remote sensing survey data sets, and comprehensively updated the new China. Special data on island coastal zone since its establishment. Through the investigation of "sky and land" integration, the distribution of resources in coastal islands and coastal zones in China was found out. The scale of satellite remote sensing atlas reached more than $1: 50,000$, and that of aerial remote sensing atlas reached 1:10,000.During the "Ninth Five-Year Plan" period, under the auspices of the 863 Program of the Ministry of Science and Technology, the Shanghai Institute of Optics and Fine Mechanics and the Institute of Marine Surveying and Mapping of the Chinese Academy of Sciences conducted airborne laser sounding system research(Li et al.,2008). In 2004, the system prototype was completed in the South China Sea. Tests show that the maximum detection depth is up to $50 \mathrm{~m}$, the sounding accuracy is positive and negative $0.3 \mathrm{~m}$, and the measurement repetition frequency is $1 \mathrm{KHz}$. The lidar uses a triple-frequency Nd:YAG laser to emit a $355 \mathrm{~nm}$ laser as the detection wavelength, and uses a linear array muti-channel photo multiplier tube array as a photoelectric conversion device. On October 16, 2009, the flight experiment of the lidar system was carried out in the Yellow Sea (Chang et al.,2002). The material fluorescence and seawater Ramon scattering signals in the sea surface and surface water were obtained, and the oil spill was judged based on the detected spectrum. In 2013, with the support of the Ministry of Science and Technology, the institute also successfully developed a prototype of the airborne dual-frequency laser lidar system, which consists of two lasers, terrestrial mapping and marine mapping. The marine mapping laser is the core of the system. Dual-wavelength lasers are used to measure the reflected signals on the sea surface and the sea floor respectively; the repetition frequency is $1 \mathrm{KHz}$ and the maximum measurement depth is $50 \mathrm{~m}$ (Rong et al.,2005). The seawater has a transmission window in the blue-green light band. The visible light in the window has a certain seawater penetration capability. The lidar detection technology uses this through-window to emit blue-green from the air platform to the seawater. The latest Mapper 5000 system has completed 11 airborne flight tests in the South China Sea to obtain three-dimensional topographic data of island reefs in the South China Sea, as shown in Figure 3.

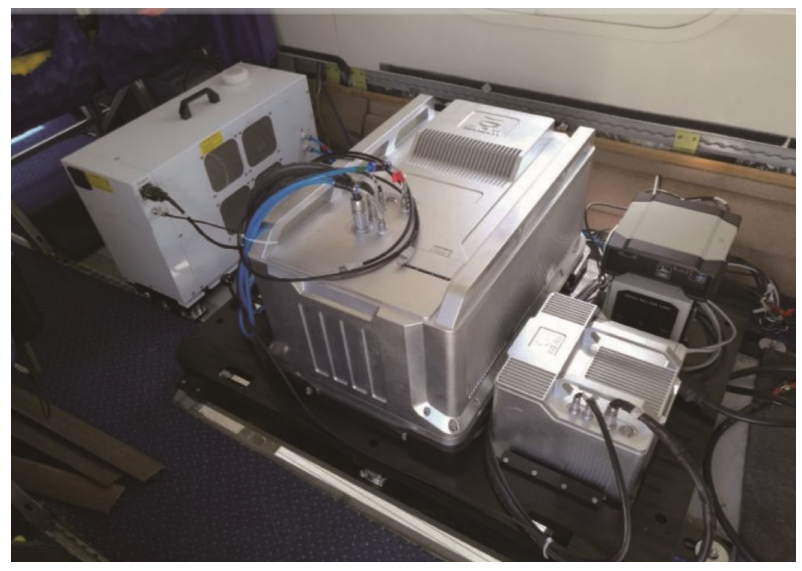

Figure 3. Mapper5000 System of Shanghai Institute of Optics and Machinery (He et al.,2018) 
With the implementation of China's latest strategy, the importance of shoreline and island reef mapping is becoming more and more important. Laser remote sensing with airborne dual-frequency lidar detection technology as its starting point will rely on its irreplaceable advantages, playing a more important role in the field of ocean exploration.

Table 1. Technical performance indicators of airborne laser sounding system recently developed in China (He et al.,2018)

\begin{tabular}{|c|c|}
\hline parameter & index \\
\hline repeat frequency & $1000 \mathrm{~Hz}$ \\
\hline Sounding range & $0.25-51 \mathrm{~m}$ \\
\hline Ocean Point Density & $1.1 \mathrm{~m} * 1.1 \mathrm{~m}$ \\
\hline Flight altitude & $250-480 \mathrm{~m}$ \\
\hline Speed & $200-245 \mathrm{~km} / \mathrm{h}$ \\
\hline Scan width & $250 \mathrm{~m}$ \\
\hline Accuracy of sounding & $0.23 \mathrm{~m}$ \\
\hline Horizontal Position Accuracy & $0.26 \mathrm{~m}$ \\
\hline
\end{tabular}

\section{CONCLUSIONS}

Although China's research on this technology is decades later than developed countries such as the United States, marine laser remote sensing technology is in a period of rapid development.

\subsection{Problems in the development of marine airborne lidar}

(1) Due to the influence of seawater quality and marine life, factors such as fish stocks and other floating objects, seawater quality, and seafloor sediments need to be considered when using the airborne laser sounding system. Therefore, in some rivers, because the water quality is too turbid, the equipment cannot be used; the seabed is mainly a muddy environment, and the mud will absorb some of the light signals, and the signal attenuation is very serious, and sometimes the system application is not ideal.

(2) A large part of the laser hitting the sea surface is absorbed and scattered by the seawater, resulting in limited measurement depth. At present, it is impossible to replace the sonar to complete the deep-sea exploration experiment.

(3) The energy attenuation of the laser from the atmosphere to the sea surface and water is too large, the underwater target echo signal is very weak, and the ocean background noise is large, which directly affects the data processing, and the sounding accuracy is very low.

(4) Sometimes affected by bad weather, large waves on the sea surface will seriously affect the laser echo signal. Moreover, due to the on-board equipment, the laser source carried by the aircraft will also be affected by the gust of wind and cause jitter, which will also affect the accuracy.

(5) Due to the high price of lidar equipment, a photoelectric converter will cost a lot of money, there is no way to mass production, and the efficiency of photoelectric conversion is too low, about $30 \%$. There is no way to satisfy some high-precision detection activities.

(6) Laser performance and data acquisition efficiency have yet to be further improved. As users' demand for high-density point clouds in large areas continues to expand, it is necessary to ensure high data collection efficiency when acquiring high-density point clouds. Due to laser diffraction limit limitations, laser detection capabilities are now approaching the detection limit.

(7) High-performance, low-cost, and compact lidar systems are in urgent demand. Large-scale lidar equipment is bound to be carried on large platforms. The large unmanned aerial vehicles and manned aircraft required for airborne lidar are too expensive. At the same time, the small lidar system is limited by the multi-rotor drone's endurance and its own performance. The time is short and the data collection efficiency is not high. (8) In terms of the maturity of data post-processing software, the current point cloud data processing is still in the development stage; especially the customized software for end users to meet the industrial application requirements still has many problems.

\subsection{Prospects for the future}

At present, lidar hardware technology is developing rapidly, industry applications are expanding and deepening, and marine laser remote sensing technology is in a stage of rapid development. In specific research and applications, the key points to note are:

(1) Further improve the positioning accuracy and depth measurement accuracy of underwater targets, improve the sounding ability of the system, especially the sounding ability of the system during clear daytime, and improve the underwater target recognition ability. Further reduce the size, weight and power consumption of the system, improve the interface between the autopilot and the system, provide a clear and simple task routing software interface, improve the operational performance of the system, and make the system practical.

(2) Innovative applied research. At present, lidar data is mainly used to produce basic mapping products and simple three-dimensional products, which have mature technologies, methods, processes and specifications. Therefore, many production units cannot agree with the advanced nature of lidar technology, which is the key to the urgent need for breakthrough. The notable direction is based on the three-dimensional change detection of lidar data and DEM data If there is a breakthrough, it will bring great progress to the application of lidar technology.

(3) In the aspect of data processing, further explore more intelligent filtering methods, improve photoelectric conversion efficiency, and more accurately determine the algorithm of underwater and water surface reflected echo signals, effectively solve the problem of signal offset and false signal recognition caused by turbid seawater. Improve the depth measurement accuracy and ability of the system.

(4) The traditional orthophoto correction technology of remote sensing image based on ground image processing platform cannot meet the requirements of real-time or near real-time. The ortho-rectification technology of (FPGA) platform is needed to solve the problem (Zhou et al.,2017).

\section{ACKNOWLEDGEMENTS}

This paper is financially supported by the National Natural Science of China under Grant numbers 41431179,41961065 ; Guangxi Innovative Development Grand Grant under the grant number: GuikeAA18118038, GuikeAA18242048; the National Key Research and Development Program of China under Grant numbers 2016YFB0502501 and the BaGuiScholars program of Guangxi (Guoqing Zhou).

\section{REFERENCES}

Burrows, W. G..,2011, Remote sensing of the environment. Science Press. 
Chen C., Wang L.,2010. 40 years of independent exploration road -- the exploration process of laser inertial confinement fusion of Shanghai Institute of Optics and precision machinery, Chinese Academy of Sciences, physics.39(7):495-502.

Chang Y., Zhu G., Peng F., Zhu Y., 2002, Survey of Airborne Lidar for Bathymetric measurement, marine sciences.26(5):34-36.

Chen W., Huang T., Lu Y., 1998, Airborne Ocean Overview of the development of lidar, laser technology.22 (3): 147-152.

Chen, F. S., Yang, K. T., \& Kang, M. W. ,2006, Polarization detection technology of underwater targets for airborne lidar. International Symposium on Advanced Optical Manufacturing \& Testing Technologies: Optical Test \& Measurement Technology \& Equipment.

Doneus M, Miholjek I, Mandlburger G, et al., 2015, Airborne Laser Bathymetry for Documentation of Submerged archaeological sites in shallow water. Underwater $3 \mathrm{~d}$ Recording \& Modelling.

Gordon A. ,1992, Use of laser scanning system on mobile underwater platforms. Symposium on Autonomous Underwater Vehicle Technology, Auv. IEEE.

He Y., Hu S., Chen W., Zhu X., Wang Y., Yang Z., Zhu X., Lü D., Yu J., Huang T., Xi X., Qu S., Yao B. ,2018, Research Progress of Domestic Airborne Dual-Frequency LiDAR Detection Technology. Laser \& Optoelectronics Progress, 55(8): 082801.

Li D., 2000, Present Situation and Development Trend of Photogrammetry in Remote Sensing, Journal of Wuhan University of Surveying and Mapping Science and Technology .25 (1): 1-6.

Lee J., Zhan H., Liu D., 2010, Research progress on polarization information in remote sensing telemetry -Spectroscopy and spectral analysis.30 (4).

Li Z., Deng J., Zhou W.,2008, Underwater Laser Detection Technology and Progress. Ship Electronic Engineering, 28 (12): 13-16+53.

Li Y., Zhong X., Yang Chao, et al., 2008, Underwater Laser Target Detection and Development. Optical Communication Technology ,32 (6): 62-65.

Penny M F, Billard B, Abbot R H. ,1989, International Journal of Remote Sensing, 10 (9) :1463-1497.

Rong J., Hu W., Zhong X.,2005, ATP tracking accuracy and the best signal light emission angle. China Laser, 32 (2): 221-223.

Ren L., Yu C., Yang Y., Liu C., 2015.An Equation of Magnetic Force Line for a Uniformed Magnetization Sphere. Marine Surveying and Mapping, 25(5),6-7.

Steinvall O,Koppari K.and Karlsson U. ,1998,Experimental evaluation of an airborne depth sounding lidar for Remote Sensing, 1714:108-126.

Von Baughan., 1995, U.S. Navy "Slide" Airborne Laser Mine Detection System, Modern Weapons (6): 30-31.
Yang J., Zhou G., Yang X.,2011,Design and Implementation of Power Supply of High-Power Diode Laser of LiDAR Onboard UAV, International Symposium on Image \& Data Fusion.

Zhou G. Zhou X.,2018, Principle, technology and application of area lidar imaging. Wuhan University Press,1 (1): 185-216.

Zhou G., Jezek, K., Wright W., Rand J., \& Granger J.,2002, Orthorectification of $1960 \mathrm{~s}$ satellite photographs covering Greenland. IEEE Transactions on Geoscience and Remote Sensing, 40(6): 1247-1259.

Zhao C., Li X., Ma Y.,2010, Preliminary analysis of airborne experiments of multi-channel ocean lidar. Papers of the 2010 Optical Congress of the Chinese Optical Society.

Zhou G., Zhang R., Li N., Huang J., Zhou X., 2017, On-board ortho-rectification for images based on an FPGA. Remote Sensing, 9(9): 874-.

Zhang Z., Ma Y., Zhang J., Liang J., Zhang J.,2015, Research on Lidar bathymetric detection model based on water echo signal simulation. Journal of Ocean Technology, 34 (6):13-18. 